\title{
Neural representations of transitive relations predict current and future math calculation skills in children
}

Flora Schwartz ${ }^{1}$, Justine Epinat-Duclos ${ }^{2}$, Jessica Léone ${ }^{2}$, Alice Poisson ${ }^{3}$ \& Jérôme Prado ${ }^{2}$

${ }^{1}$ Department of Psychiatry and Behavioral Sciences, Stanford University, USA

${ }^{2}$ Lyon Neuroscience Research Center (CRNL), Experiential Neuroscience and Mental Training Team, INSERM U1028 - CNRS UMR5292, University of Lyon, France

${ }^{3}$ GénoPsy, Reference center for rare diseases with psychiatric symptoms, Centre Hospitalier le Vinatier, France

Correspondence should be addressed to F.S. (florasch@stanford.edu) or J.P. (jerome.prado@univ-lyon1.fr), Lyon Neuroscience Research Center, CH Le Vinatier, 95 bd Pinel, 69675 Bron Cedex, France. Phone: +33 (0)4 37911265. 


\begin{abstract}
A large body of evidence suggests that math learning in children is built upon innate mechanisms for representing numerical quantities in the intraparietal sulcus (IPS). Learning math, however, is about more than processing quantitative information. It is also about understanding relations between quantities and making inferences based on these relations. Consistent with this idea, recent behavioral studies suggest that the ability to process transitive relations $(\mathrm{A}>\mathrm{B}, \mathrm{B}>\mathrm{C}$, therefore $\mathrm{A}>\mathrm{C}$ ) may contribute to math skills in children. Here we used fMRI coupled with a longitudinal design to determine whether the neural processing of transitive relations in children could predict their current and future math skills. At baseline $\left(T_{1}\right)$, children $(n=31)$ processed transitive relations in an MRI scanner. Math skills were measured at $T_{1}$ and again 1.5 years later $\left(T_{2}\right)$. Using a machine learning approach with cross-validation, we found that activity associated with the representation of transitive relations in the IPS predicted math calculation skills at both $\mathrm{T}_{1}$ and $\mathrm{T}_{2}$. Our study highlights the potential of neurobiological measures of transitive reasoning for forecasting math skills in children, providing additional evidence for a link between this type of reasoning and math learning.
\end{abstract}

Keywords: math learning, transitive reasoning, arithmetic, neuromarker, fMRI 


\section{Introduction}

The ability to understand and manipulate logical relations has long been thought to be associated with the acquisition of math skills in children. For instance, the pioneering developmental psychologist Jean Piaget famously proposed that the development of math cognition relies on the emergence of logical skills from childhood to adolescence (Piaget 1952). Educational policies have also often been influenced by the idea that learning math would contribute to the development of logical thinking in children, a claim whose premises can be found in the writings of philosophers such as Plato, Locke, and Bacon (Inglis and Attridge 2017). To date, however, evidence that math learning is related to logical reasoning remains scarce, most likely because research has largely focused on investigating to what extent math development relies on evolutionally old mechanisms for representing quantities (Feigenson, Dehaene et al. 2004).

Yet, mathematical development is about more than simply processing quantitative information. It is also about understanding relations between those quantities and making inferences based on these relations. For example, a type of logical relation that is prevalent in many math domains is that of transitivity. Transitivity is a property that arises from a set of items that can be ordered along a single continuum (Wright 2001). A relation is said "transitive" when it allows reasoners to infer a relationship between two items (e.g., A > C) from two other overlapping pairs (e.g., A > B; B > C). The ability to recognize transitive relations and make associated inferences may contribute to the acquisition of many mathematical concepts. For example, transitive reasoning is fundamental to the acquisition of measurement skills in children (Inhelder and Piaget 1958, Bryant and Kopytynska 1976, Rabinowitz and Howe 1994). It also allows for the processing of ordinal and categorical information, which in turn contributes to domains such as arithmetic, algebra and geometry (Bryant and Kopytynska 1976, Rabinowitz and Howe 1994, Wright 2001). 
Consistent with a role for transitive reasoning in math learning, a few studies have shown that the ability to understand transitive relations relate to math skills in children. For example, Morsanyi, Devine et al. (2013) found that 10-year-olds with math learning disability (i.e., dyscalculia) performed significantly worse than typically developing controls in a transitive reasoning task. In contrast, children with high math ability performed significantly better than typically developing control on that same task. This is in line with a prior report showing that math performance is generally related to transitive (as well as conditional) reasoning ability in elementary school children (Handley, Capon et al. 2004).

What neural mechanisms may underlie the relationship between transitive reasoning and math learning in children? Two lines of evidence suggest that this relationship may be mediated by brain mechanisms involved in relational processing in the posterior parietal cortex (Wendelken 2015). First, neuroimaging studies indicate that the processing of transitive relations consistently activates the intraparietal sulcus (IPS) (for a review, see Prado, Chadha et al., 2011), a region that is also typically involved in tasks that require the manipulation of numbers (Nieder and Dehaene 2009). Arguably, both transitive reasoning and numerical cognition require individuals to process ordinal information. Therefore, this common reliance on IPS mechanisms may stem from the fact that this region is central to ordinal processing more generally, in line with studies showing that the IPS is also activated when participants represent learned series such as letters, days or months (Fias, Lammertyn et al. 2007).

However, it is also possible that transitive relations and numbers are encoded in similar regions of the IPS because they both rely on representations that are inherently spatial and for which the posterior parietal cortex is key (Prado, Noveck et al. 2010). For example, numbers are often thought to be represented on a mental number line (Hubbard, Piazza et al. 2005) in adults and children. This is notably suggested by the behavioral distance effect 
observed in number comparison tasks (i.e., reaction times decrease with the distance between numbers; Moyer and Landauer 1967), indicating that numbers that are close (e.g., 4 vs 5) are more difficult to distinguish than numbers that are far (e.g., 2 vs 8 ). This phenomenon has also been reported in transitive reasoning (i.e., reaction times decrease with the distance between items in a transitive ordering; Prado, Noveck et al. 2010), suggesting that transitive items are also arranged on a mental representation of space (Alfred, Connolly et al. 2018). Furthermore, activity in same region of the IPS has been found to decrease with the distance between numbers (Pinel, Dehaene et al. 2001, Pinel, Piazza et al. 2004, Mussolin, Mejias et al. 2010) or transitive items (Prado, Noveck et al. 2010), suggesting that transitive relations and numbers engage common spatial representations in the IPS.

A second line of evidence suggesting that the relationship between transitive reasoning and math learning may be mediated by the posterior parietal cortex is provided by a recent neuroimaging study. In that study, we contrasted performance and brain activity of typically developing children to children with math learning difficulties during a transitive reasoning task. While transitive relations were associated with IPS activity in typically developing children, that was not the case in children with math learning difficulty (Schwartz, EpinatDuclos et al. 2018). Children with math learning difficulty also showed significantly less activity in the IPS than typically-developing children during the transitive reasoning task. Thus, the extent to which IPS mechanisms process transitive relations may be related to levels of math competence in children.

It is important to note that our previous study only provides indirect correlational evidence that math abilities may be associated with reasoning-related activity in the IPS. However, it raises an intriguing possibility that may strengthen the claim that transitive reasoning plays an important role in math learning: current and future math skills in children might be predicted (at least to some extent) by the neural processing of transitive relations in 
the IPS. The goal of the present study was to formally test this hypothesis. FMRI activity of 31 typically-developing children from 9 to 13 was measured while they passively listened to transitive (e.g., $\mathrm{A}>\mathrm{B}, \mathrm{B}>\mathrm{C}$ ) and non-transitive (e.g., $\mathrm{A}>\mathrm{B}, \mathrm{C}>\mathrm{D}$ ) relations that were embedded in a coherent story that was designed to be as interactive (it took the form of a "choose your own adventure story") and as engaging as possible (Schwartz, Epinat-Duclos et al. 2018). This task allowed us to measure activity associated with transitive relation in a relatively ecological context (i.e., discourse comprehension). Arithmetic and math problemsolving abilities were measured for each child at the time of the fMRI session $\left(T_{1}\right)$, as well as 1.5 years later $\left(\mathrm{T}_{2}\right)$. The predictive power of the neural representations of transitive relations in the IPS on current and future math skills was assessed using a machine learning approach with cross-validation (Gabrieli, Ghosh et al. 2015). Specifically, we evaluated whether multivariate patterns of IPS activity associated with the processing of transitive relations (as compared to non-transitive relations) at $\mathrm{T}_{1}$ could accurately predict math scores of children at both $\mathrm{T}_{1}$ and $\mathrm{T}_{2}$.

\section{Material and methods}

\section{Sample size justification}

To our knowledge, this is the first study to examine the link between individual differences in math skills and the neural processing of transitive relations in typically developing children. For this reason, and because decoding accuracy in multivariate analyses may not reflect effect sizes (Hebart and Baker 2018), it is difficult to determine the optimal sample size for the multivariate analyses. However, we note that previous studies that have used multivariate analyses to predict math skills in children have used sample sizes ranging from $n=20$ (Evans, Kochalka et al. 2015) to $n=28$ (Qin, Cho et al. 2015). Power 
calculations (performed with G*Power, https://stats.idre.ucla.edu/other/gpower/) indicate that such sample sizes would provide $80 \%$ power to detect a univariate brain-behavior correlation in the $r=0.5$ to $r=0.6$ range (with $\alpha=0.05$ ). Such a range is typically the one observed in most studies measuring univariate brain-behavior correlations (providing that regions in which correlations are observed are not selected from circular analyses; see Figure 5 in Vul, Harris et al. 2009). Therefore, we aimed to analyze a sample size of around 28 participants in the present study (similar to Qin, Cho et al. (2015). Because we expected to discard about 20\% of participants due to technical, performance, or movement issues, we aimed to recruit around 35 participants.

\section{Participants}

Thirty-eight right-handed children from 8 to 13 were recruited using advertisements in schools, newspapers and social media. Seven of these participants were excluded from the analyses because of excessive head motion in at least 3 of the 4 fMRI runs $(n=4)$, presence of a specific language impairment $(\mathrm{n}=1)$, and lack of behavioral data $(\mathrm{n}=2)$. Thus, 31 children were included in the analyses. Eighteen of these participants were already analyzed in Schwartz, Epinat-Duclos et al. (2018), in which only univariate analyses were used at $\mathrm{T}_{1}$ to compare brain activity associated with transitive reasoning between children with and without math learning difficulty. The mean age of the final sample was 10.97 years $(S D=1.37)$ at $\mathrm{T}_{1}$ and 12.57 years $(S D=1.39)$ at $\mathrm{T}_{2}$. All children were native French speakers and had no diagnosis of mental retardation or high intellectual potential. They also had no hearing deficit, no MRI contraindications and no history of neurological and psychiatric disorder. Parents gave their written informed consent and children gave their assent to participate in the experiment, which was approved by the local ethics committee (CPP Lyon Sud-Est II). Families were paid 80 euros for their participation. 


\section{Testing}

To ensure that participants had normal cognitive functioning and to provide a means of measuring math skill, children completed standardized tests at the time of scanning $\left(T_{1}\right)$ and after a follow-up period of between 1.45 and 1.79 years $\left(\mathrm{T}_{2}\right)($ mean $=1.60$ years, $S D=$ 0.07). First, the Nouvelle Echelle Metrique de l'Intelligence (NEMI-2) (Cognet 2006) was used to obtain a measure of verbal intelligence (estimated using subtests of general knowledge, vocabulary and comparisons) and matrix reasoning at $\mathrm{T}_{1}$. Second, math skills were measured at both $\mathrm{T}_{1}$ and $\mathrm{T}_{2}$ using a French version of the Woodcock-Johnson Test of achievement (WJ III; Woodcock, McGrew et al., 2001). To provide a comprehensive measure of math abilities in each individual, 3 subtests were used: Calculation, Math fluency, and Applied problems. The Calculation subtest is an untimed test in which participants perform math computations of increasing difficulty. The test begins with single-digit addition, subtraction and multiplication problems and progressively moves to double-digit problems (including division) and algebra, as well as logarithmic and trigonometric operations. Test administration is stopped after six consecutive errors or when the last item is reached (raw scores range from 0 to 45 ). In the Math fluency subtest, participants have 3 minutes to solve as many single-digit addition, subtraction and multiplication problems (raw scores range from 0 to 160 ). The Applied problems subtest measures the ability to analyze and solve math problems. While early items require application of basic numerical concepts (e.g., counting, performing simple addition and subtraction, reading clocks and coin values), most items require children to understand and analyze word problems. The test is untimed and testing stops after 6 consecutive errors or when the last item is reached (raw scores range from 0 to 63). Standardized IQ scores at $T_{1}$ and raw math scores at $T_{1}$ and $T_{2}$ are shown in Table 1. 
Participants completed the task described in Schwartz, Epinat-Duclos et al. (2018). In the scanner, they listened to 4 stories that each included a series of 12 short scenarios (see Table 2). Each scenario ended up with a question that the child had to answer. Similar to a "choose your own story" structure, children were told to pay attention to every scenario because their responses to questions were critical for making progress in the story. Scenarios varied with respect to the type of relations involved. Twenty-four scenarios included linear-order relations such as "A is more than B", while 24 scenarios included set-inclusion relations such as "All As are Bs". In half (i.e., 12) of each type of scenario, the relations overlapped so that they could be integrated, and a conclusion could be inferred. For instance, the linear-order relations "White cows give more milk than black cows" and "Black cows give more milk than brown cows" in the bottom left cell of Table 2 should lead to the conclusion that "White cows give more milk than brown cows". Similarly, the set-inclusion relations "All old farms are made of stone" and "All farms that are made of stone are uphill" in the top left cell of Table 2 should lead to the conclusion that "All old farms are uphill". The question that followed scenarios with such transitive relations always tested whether children had inferred conclusions upon listening to the scenario (it was termed Reasoning question). The remaining scenarios included nontransitive relations, that is, relations that were not overlapping. Thus, no particular conclusion could be inferred upon listening to the scenario. For instance, the linear-order relations "The chocolate cake is baking faster than the apple pie" and "The strawberry pie is baking faster than the cheesecake" in the bottom right cell of Table 2 are non-transitive. Similarly, the setinclusion relations "All bedrooms with a red door are on the side of the chicken coop" and "All bedrooms with a green door are on the side of the barn" in the top right cell of Table 2 are nontransitive. In those cases, the question that followed simply tested whether children remembered 
some information that was explicitly given in the scenario and was termed Memory question (see Table 2).

Overall word count was controlled across the 4 types of scenarios. On average, word count was 69.1 for scenarios with transitive set-inclusion relations, 69.7 for scenarios with nontransitive set-inclusion relations, 73.6 for scenarios with transitive linear-order relations and 72.4 for scenarios with non-transitive linear-order relations. Because word count was not normally distributed, non-parametric testing was used to assess differences as a function of type of scenarios. A Kruskal-Wallis ANOVA indicated that word count did not differ between types of scenarios $\left(H_{(3,48)}=4.14, p=0.25\right)$.

The task was split into 4 runs that contained one story each. Each story contained 3 scenarios with transitive linear-order relations, 3 scenarios with non- transitive linear-order relations, 3 scenarios with transitive set-inclusion relations and 3 scenarios with nontransitive set-inclusion relations. Two wrap-up sentences concluded each story. Runs were randomized but scenarios were presented in a fixed order within a run. This was because scenarios were embedded in a coherent story. However, two scenarios of the same type were never following each other. Additionally, responses were counterbalanced across different variables. First, the order of the correct response was counterbalanced between the 4 types of stories across the whole experiment. Second, within a run, the order of the correct response was counterbalanced between scenarios with transitive and non-transitive relations and between scenarios involving linear-order relations and set-inclusion relations. Third, to prevent participants from developing expectations during the task and using heuristic strategies to respond to Reasoning questions, transitive relations were switched around in half of the problems. For example, linear-order relations could be presented in the order " $\mathrm{A}$ is faster than B and B is faster than C" in some scenarios and in the order "B is faster than $\mathrm{C}$ and A is faster than B" in other scenarios. Participants were instructed to press on a response 
button when they were ready to hear the next sentence. They were told to press on one of two other response buttons to choose between options in the question. Each story started with a red fixation cross at the center of the screen. The red cross turned orange after $6 \mathrm{~s}$ and green after $2 \mathrm{~s}$. The green cross lasted $2 \mathrm{~s}$ and was immediately followed by the presentation of the first sentence of the first scenario. There was a $500 \mathrm{~ms}$ interval between each sentence in a scenario. A white fixation cross was displayed before and after each question for a random duration between 2 and $4 \mathrm{~s}$. A fixation cross was also displayed for $20 \mathrm{~s}$ at the end of each story. See Figure 1 for a timeline of one scenario.

\section{Experimental procedure}

After standardized tests were administered at $\mathrm{T}_{1}$, children were familiarized with the fMRI environment in a mock scanner. They listened to a recording of the noises associated with all fMRI sequences. A motion tracker system (3D Guidance trak STAR, Ascension Technology Corporation) was used to measure head movements and provide on-line feedback to participants. Finally, children practiced the task in that mock scanner. Different stimuli were used in the practice and in the scanning sessions. Stimuli were generated using Presentation software (Neurobehavioral Systems, Albany, CA). Stories were spoken through headphones sentence by sentence. During each scenario, a black-and-white picture illustrating the setting was displayed on a computer screen that was viewed by the participants through a mirror attached to the head coil. Behavioral responses were recorded using MR-compatible keypads placed in the left and right hands.

\section{fMRI: data acquisition}

Images were collected with a Siemens Prisma 3T MRI scanner (Siemens Healthcare, Erlangen, Germany) at the CERMEP Imagerie du vivant in Lyon, France. The BOLD signal 
was measured with a susceptibility weighted single-shot Echo-planar Imaging (EPI) sequence. Imaging parameters were as follows: $\mathrm{TR}=2000 \mathrm{~ms}, \mathrm{TE}=24 \mathrm{~ms}$, flip angle $=80^{\circ}$, matrix size $=128 \times 120$, field of view $=220 \times 206 \mathrm{~mm}$, voxel size $=1.72 \times 1.72 \mathrm{~mm}$, slice thickness $=3$ $\mathrm{mm}(0.48 \mathrm{~mm}$ gap $)$, number of slices $=32$. A high-resolution T1-weighted whole-brain anatomical volume was also collected for each participant. Parameters were as follows: TR = $3500 \mathrm{~ms}, \mathrm{TE}=2.24 \mathrm{~ms}$, flip angle $=8^{\circ}$, matrix size $=256 \times 256$, field of view $=224 \times 224$ $\mathrm{mm}$, voxel size $=0.87 \times 0.87 \mathrm{~mm}$, slice thickness $=0.9 \mathrm{~mm}$, number of slices $=192 . "$

\section{fMRI: data preprocessing}

Images were analyzed with SPM12 (Welcome department of Cognitive Neurology, London, UK). The first 4 images of each run were discarded to allow for T1 equilibration effects. Functional images were corrected for slice acquisition delays and spatially realigned to the first image of the first run to correct for head movements. Realigned images were smoothed with a Gaussian filter ( 4 x 4 x 8 mm full-width at half maximum). As in our previous studies (Schwartz, Epinat-Duclos et al. 2017, Mathieu, Epinat-Duclos et al. 2018, Schwartz, Epinat-Duclos et al. 2018, Schwartz, Epinat-Duclos et al. 2018), volumes showing rapid scan-to-scan movement greater than $1.5 \mathrm{~mm}$ were identified using ArtRepair (Mazaika, Hoeft et al. 2009). Those were removed and replaced by the interpolation of the 2 nearest non-repaired volumes. Runs with more than $10 \%$ of repaired volumes were excluded from the analysis. Finally, functional images were normalized into the standard Montreal Neurological Institute (MNI) space.

\section{fMRI: statistical modeling}

Statistical modeling was performed in the context of the General Linear Model, using the exact same procedure as in Schwartz, Epinat-Duclos et al. (2018). Sentences of interest 
were those containing transitive and non-transitive relations (i.e., sentences 4 and 5). These sentences were modeled as epochs with onsets time-locked to the presentation of sentence 4 and with offsets time-locked to the end of sentence 6 (i.e., the wrap-up sentence). Other sentences (as well as the question) were not explicitly modeled and were considered background noise. Linear-order and set-inclusion relations were modeled within the same regressors. All epochs were convolved with a canonical hemodynamic response function (HRF). The time series data were high-pass filtered $(1 / 128 \mathrm{~Hz})$, and serial correlations were corrected using an autoregressive AR(1) model.

\section{fMRI: ROIs definition}

Regions of interest (ROIs) were regions in which greater activity was observed for transitive than non-transitive relations. The definition of these regions involved the following 3 steps. First, for each subject, brain activity associated with non-transitive relations was subtracted from brain activity associated with transitive relations across the whole-brain. Second, these contrast images (one for each participant) were submitted to a second-level onesample t-test. Third, the resulting t-map was thresholded using the non-parametric permutationbased Threshold-Free Cluster Enhancement (TFCE) method (Smith and Nichols 2009), implemented in the TFCE Toolbox r164 (http://dbm.neuro.uni-jena.de/tfce/). As in Schwartz, Epinat-Duclos et al. (2018), we had an a priori hypothesis that transitive relations would be

processed in the IPS. Therefore, clusters were considered significant at a Family-Wise Error(FWE) corrected threshold of $p<0.05$, either across the whole-brain or within an anatomical mask of the IPS (i.e., small volume correction) defined using the Anatomy Toolbox v2.2 (Eickhoff, Stephan et al. 2005). Following Schwartz, Epinat-Duclos et al. (2018), (Schwartz, Epinat-Duclos et al. 2018)the IPS mask consisted in voxels with at least 50\% probability of belonging to one of the IPS subdivisions (hIP1, hIP2, and hIP3) as defined in the Anatomy 
Toolbox (Choi, Zilles et al. 2006, Scheperjans, Eickhoff et al. 2008, Scheperjans, Hermann et al. 2008).

\section{fMRI: ROI analysis}

Activity in the ROIs defined using the procedure above was analyzed using both univariate and multivariate methods. First, we calculated for each participant the average activity associated with the contrast of transitive versus non-transitive relations within an ROI by averaging the fMRI signal across all voxels within that ROI. This average activity was then correlated with Calculation, Math fluency, and Applied problems raw scores at $T_{1}$ and $T_{2}$ across participants.

Second, because such standard univariate regression analyses only show associations between two within-sample variables but do not have any predictive power (Gabrieli, Ghosh et al. 2015), we used a multivariate machine-learning approach with cross-validation to test whether brain activity associated with the contrast of transitive versus non-transitive relations could predict math scores of out-of-sample participants at both T1 and T2. This was done using the pipeline for pattern regression analyses provided by the Pattern Recognition for Neuroimaging Toolbox (PRoNTo) v2.0 implemented in Matlab (http://www.mlnl.cs.ucl.ac.uk/pronto/) (Schrouff, Rosa et al. 2013). This pipeline involves a training phase and a test phase (Portugal, Rosa et al. 2016). During the training phase, a pattern regression model is trained by associating labels with spatial patterns of activity from a "training set". During the test phase, the association between patterns and labels (learned in the training phase) is used to predict the label of novel (i.e., out-of-sample) patterns from a "test set" (Schrouff, Rosa et al. 2013). In the present study, spatial patterns were voxel-byvoxel patterns of activity for the contrast of transitive versus non-transitive relations from each ROI. Labels were Calculation, Math fluency, and Applied problems raw scores at both 
$\mathrm{T}_{1}$ and $\mathrm{T}_{2}$. Following studies suggesting that Kernel Ridge Regression (KRR) (Shawe-Taylor and Cristianini 2004) is computationally effective and may give better prediction accuracy than other linear methods (Chu, Ni et al. 2011, Mihalik, Brudfors et al. 2019), we used KRR to predict math scores. Briefly, KRR estimates a relationship between samples by minimizing an error function that includes the sum of the squared differences between model predictions and regression targets as well as a model regularization term (Taylor, Matthews et al. 2017). Partitioning between the training and the test sets was done using a leave-one-subject-out cross-validation method. This involves leaving one participant out for test and training the model on 30 (i.e., $n-1)$ participants. The procedure was then repeated 31 times, such that each participant was left out once.

Prediction accuracy of the model was measured using the Pearson's correlation coefficient (r) and the mean squared error (MSE) of the relationship between actual and predicted scores. Positive r values indicate that the model is able to predict the outcome, while negative $r$ values (or $r$ values close to 0 ) indicate that the model fails to predict the outcome. Statistical significance was calculated using permutation tests with 1,000 iterations. That is, the value for each metric (i.e., $r$ and MSE) was compared to the results of 1,000 random permutations (representing the distribution of correlations corresponding to the null hypothesis). P values were calculated based on how many times the value obtained with random permutations was higher than the correlation value observed in the data (divided by $1,000)$. 


\section{Results}

\section{ROIs definition}

ROIs involved in transitive reasoning were identified by contrasting activity associated with transitive relations to activity associated with non-transitive relations. As shown in Table 3 and Figure 2, this contrast revealed significant activity in two clusters of the left IPS and one cluster of the right IPS. These clusters defined the voxels that were used in the subsequent univariate and multivariate analyses (see Methods). Specifically, both left IPS clusters constituted the left IPS ROI (158 voxels) and the right IPS cluster constituted the right IPS ROI (154 voxels). No region outside of the IPS survived whole-brain correction for multiple comparisons.

Relationships between math scores, transitive reasoning performance, and univariate IPS activity

Transitive reasoning performance was measured using accuracy to reasoning questions during the fMRI task at $\mathrm{T}_{1}$. Accuracy to reasoning questions ranged from 0.5 to 1 (average $=$ $0.75, S D=0.15$ ). Accuracy to memory questions ranged from 0.61 to 1 (average $=0.82, S D=$ 0.12). Table 4 contains the correlations among math scores (at T1 and T2), accuracy to reasoning and memory questions, and univariate activity associated with the contrast of transitive versus non-transitive relations in the left and right IPS ROIs (all correlations partialled out differences in age and verbal IQ between participants). Although there was no relationship between Calculation scores and accuracy to Reasoning questions, there was a significant relationship between Calculation scores and univariate activity in the left and right 
IPS, at both T1 and T2 (see Table 4). However, these brain-behavior correlations were specific to the Calculation subtest, as neither Math fluency nor Applied problem scores were correlated with univariate activity in the IPS (at T1 or T2). This was despite the fact that accuracy to reasoning questions did correlate with scores on these two subtests (but only at $\mathrm{T}_{1}$ ). Thus, univariate activity associated with the contrast of transitive versus non-transitive relations was specifically related to math calculation skills at the time of scanning and 1.5 years later.

\section{Predictive power of multivariate activity associated with transitive reasoning}

The analyses above, however, only reflect in-sample associations between brain activity during the processing of transitive relations and math abilities. As such, they do not inform on whether brain activity during the processing of transitive relations may predict the outcome of out-of-sample individuals (Gabrieli, Ghosh et al. 2015). To address this question, we used regression-based multivariate analyses with cross-validation. As in univariate analyses, age and measures of verbal IQ (general knowledge, comparison, and vocabulary) were included as confounding variables. These analyses tested whether patterns of IPS activity associated with the contrast of transitive versus non-transitive relations could accurately predict current math score in out-of-sample individuals, over and above differences in age and verbal IQ (see Methods). Accuracy of the machine learning algorithm was assessed by examining the relationship between actual and predicted math scores at $T_{1}$. As shown in Figure 3 (left), there was no relationship between actual and predicted Calculation scores in the left IPS at T1 $(r=-0.07, p=0.60 ;$ MSE $=38.05, p=0.64)$ or T2 $(r=-0.26, p=$ 0.87 MSE $=92.77, p=0.89)$. However, the relationship was reliable in the right IPS at both $\mathrm{T} 1(r=0.60, p=0.002 ; \mathrm{MSE}=11.87, p=0.006)$ and T2 $(r=0.39, p=0.04 ; \mathrm{MSE}=23.92, p$ 
$=0.01)$. Figure 4 shows the consistency between the actual and the predicted Calculation score for each participant in the left and right IPS at both $\mathrm{T}_{1}$ and $\mathrm{T}_{2}$. In contrast to Calculation scores, there was no reliable relationship between actual and predicted Math fluency scores at either $\mathrm{T}_{1}$ or $\mathrm{T}_{2}$ (all $r s<0.11$, all $p s>0.32$; all MSEs $>466.17$, all $\left.p s>0.30\right)$. Similarly, there was no reliable relationship between actual and predicted Applied problem scores at either $\mathrm{T}_{1}$ or $\mathrm{T}_{2}($ all $r s<0.06$, all $p s>0.39$; all MSEs $>54.31$, all $p s>0.36)$. Consistencies between actual and predicted Math fluency and Applied problem scores are shown in Supplementary Figure 1 and Supplementary Figure 2. Therefore, patterns of right IPS activity associated with transitive relations predicted math calculation skills (but not arithmetic fluency or problem-solving skills) at the time of scanning and 1.5 years later.

\section{Discussion}

Over the past decades, developmental research on math learning has largely focused on exploring the role of mechanisms for representing numerical quantities (Ansari 2008). Yet, acquiring math skills also involves understanding relations between quantities and drawing inferences based on these relations (Singley and Bunge 2014). In keeping with this idea, recent behavioral studies suggest that understanding relations of transitivity may significantly contribute to math learning in children (Handley, Capon et al. 2004, Morsanyi, Devine et al. 2013, Morsanyi, Kahl et al. 2017, Morsanyi, McCormack et al. 2017). Here we tested whether the brain representations of transitive relations may predict current and future math skills in children. Specifically, fMRI activity was measured while children listened to transitive and non-transitive relations embedded in a story context. Calculation, single-digit arithmetic, and problem-solving skills were also measured at the time of scanning $\left(\mathrm{T}_{1}\right)$ and 18 months later $\left(\mathrm{T}_{2}\right)$. A machine learning approach with cross validation was used to test whether activity 
associated with the contrast of transitive versus non-transitive relations could predict math skills at both $\mathrm{T}_{1}$ and $\mathrm{T}_{2}$.

First, we found that transitive relations were associated with greater activity than nontransitive relations in the IPS. This is consistent with several previous neuroimaging studies that have also found that transitive reasoning mainly relies on regions in and around the IPS (Goel and Dolan 2001, Acuna, Eliassen et al. 2002, Knauff, Mulack et al. 2002, Knauff, Fangmeier et al. 2003, Heckers, Zalesak et al. 2004, Fangmeier, Knauff et al. 2006, Hinton, Dymond et al. 2010, Prado, Noveck et al. 2010, Prado, Van Der Henst et al. 2010, Prado, Mutreja et al. 2013). For example, quantitative meta-analyses of the literature show that transitive reasoning consistently activates the left and right IPS across studies in adults (Prado, Chadha et al. 2011, Wertheim and Ragni 2018). A recent study in children also points to the involvement of the parietal cortex in transitive reasoning (Mathieu, Booth et al. 2015). Thus, together with these previous studies, our results suggest that the IPS houses mechanisms that are important for representing and processing transitive relations. This may be because transitive relations are mapped onto spatial mental models (Goodwin and JohnsonLaird 2005) that are encoded and represented in the IPS (Alfred, Connolly et al. 2018).

Second, a major novel finding from the present study is that IPS activity associated with the contrast of transitive versus non-transitive relations was predictive of math skills at both $T_{1}$ and $T_{2}$. This predictive power, however, was limited to math calculation skills as measured by the Calculation subtest of the Woodcock-Johnson III battery. That is, it was not observed with the Math fluency subtest (reflecting arithmetic fluency) and the Applied problems subtest (reflecting broader problem-solving skills). Why would neural activity in the (right) IPS during the processing of transitive relations specifically predict current and future calculation skills? One possibility is that math calculation recruits mechanisms supporting the processing of transitive relations in the IPS because numerical operations are inherently 
relational (i.e., they involve relating quantities to each other). Thus, activity related to transitive reasoning in the IPS may reflect the way this region processes relational information in children (Wendelken 2015), which may in turn relate to their calculation skills. This possibility, however, is undermined by the fact that activity associated with transitive reasoning does not predict arithmetic fluency (as reflected by knowledge of single-digit problems in the Math fluency subtest). Even though they are largely thought to be retrieved from long-term memory rather than calculated (Campbell and Xue 2001), single-digit arithmetic problems are also arguably relational and should therefore be related to IPS activity is it reflects relational processing.

Another possibility, which may be more likely, is that both transitive reasoning and calculation skills may rely on a set of IPS mechanisms involved in ordering information in working-memory. The role of the IPS in processing numerical and non-numerical ordinal information has been suggested by several studies (Marshuetz 2005, Majerus, Poncelet et al. 2006). Children with math learning disability, who exhibit anatomical and functional impairment in the IPS, have also deficits when processing ordinal information (Attout and Majerus 2015, De Visscher, Szmalec et al. 2015). A prominent cognitive theory of human reasoning (i.e., the Mental Models theory) claims that integrating transitive relations relies on the representation and manipulation of ordered mental models in working memory (Goodwin and Johnson-Laird 2005). The ability to order numerical information is also fundamental to arithmetic skills (Knops and Willmes 2014, Lyons, Price et al. 2014). Therefore, it is possible that a common reliance on ordinal working memory in the IPS underlies the relationship between the processing of transitive relations and arithmetic learning. Interestingly, this possibility would account for the fact that IPS activity associated with transitive reasoning is not associated with Math fluency scores. As mentioned above, single-digit arithmetic problems are typically thought to be either directly retrieved from long-term memory or 
automatized through procedural knowledge by the end of elementary school (Thevenot, Barrouillet et al. 2016). Thus, these problems are likely to rely to a lesser extent on working memory resources and involve brain mechanisms supporting the retrieval of verbal information in the left temporo-parietal cortex, rather than right IPS mechanisms typically associated with working memory and more effortful calculation strategies (Zamarian, Ischebeck et al. 2009, Prado, Mutreja et al. 2011).

More generally, it is also noteworthy that the predictive relationship between neural activity associated with transitive reasoning and calculation skills was observed in the right rather than the left IPS. Both the right and left IPS are involved in math cognition (Sokolowski, Fias et al. 2017, Arsalidou, Pawliw-Levac et al. 2018), but a dissociation may emerge during development. It has notably been suggested that the neural mechanisms supporting math skills may develop earlier in the right than in the left IPS, notably because they are involved in non-symbolic processing (Cantlon, Brannon et al. 2006, Emerson and Cantlon 2015, Vogel, Goffin et al. 2015). Thus, although transitive reasoning has typically been associated with bilateral IPS activity (Goel and Dolan 2003, Knauff, Fangmeier et al. 2003, Fangmeier, Knauff et al. 2006, Prado, Noveck et al. 2010, Mathieu, Booth et al. 2015, Schwartz, Epinat-Duclos et al. 2018), its interaction with math processing may be more readily observed in the right than left hemisphere in children.

It is interesting to note that we did not find any relationship between IPS activity associated with transitive reasoning and Applied problems scores. One possibility is that transitive reasoning does not critically support math problem-solving skills (as measured by the Applied problems subtest) in the developmental range considered. For instance, transitive reasoning may be particularly important earlier on when acquiring foundational math concepts such as measurement, serial-order and categorization (Bryant and Kopytynska 1976, Rabinowitz and Howe 1994, Wright 2001). Although this suggests that transitive reasoning 
and math problem-solving may be observed in children younger than the age range considered here, accuracy to reasoning questions was related to score on the Applied problems subtest in our sample (at least at the time of testing). Thus, it is likely that brain activity during transitive reasoning also relates to Applied problems scores on some levels, even though this relation may be observed in brain regions that were not identified here. For instance, the Applied problems subtest involves math word problems that may rely on brain regions involved in verbal processing and executive control in the prefrontal cortex (Prabhakaran, Rypma et al. 2001, Newman, Willoughby et al. 2011). Unfortunately, we were not able to localize frontal brain regions involved in transitive reasoning at the whole-brain level. This might be investigated in future studies.

Finally, we found that both univariate and multivariate activity associated with the neural processing of transitive relations in the IPS predicted calculation skills at $T_{1}$ and $T_{2}$, even though there was no behavioral relationship between transitive reasoning performance and calculation skills (over and above differences in age and verbal IQ). As pointed out by Evans, Kochalka et al. (2015), this may indicate that neurobiological measures are more sensitive than behavioral measurements in predicting learning outcomes. For example, activity in the right inferior frontal gyrus has been found to predict long-term reading gains in children with dyslexia even though no behavioral measures of reading ability could do so (Hoeft, McCandliss et al. 2011). In the domain of math cognition, previous neuroimaging studies have found that neither measures of executive functions nor measures of numerical abilities could accurately predict short-term (Supekar, Swigart et al. 2013) or long-term (Evans, Kochalka et al. 2015) changes in math performance, whereas functional and anatomical brain measurements were predictive of learning. Therefore, our findings add to the growing evidence that brain measures have a unique potential for forecasting academic skills. 
In summary, the present longitudinal study indicates that the neural processing of transitive relations may predict current and future calculation skills in children. These results add to growing evidence that math learning does not only rely on the ability to process numerical quantities, but also critically involves the ability to understand and process logical relations (Singley and Bunge 2014). Because calculation skills were not related to transitive reasoning performance, our findings also suggest that fMRI measures may be more sensitive than behavioral measures in predicting academic skills. Overall, the present results call for future studies investigating the relationship between neurobiological measures of relational reasoning and different types of math learning in younger children as well as in children with math learning disability.

Acknowledgments: This research was supported by a grant from the Agence Nationale de la Recherche (ANR-14-CE30-0002) to J.P. We thank the Hospices Civils de Lyon for sponsoring the research, as well as Romain Mathieu, Auriane Couderc, Inès Daguet, and the MRI engineers (Franck Lamberton and Danielle Ibarrola) at the CERMEP-Lyon platform for their assistance in collecting the fMRI data.

Conflict of Interest: The authors declare no competing financial interests. 


\section{Reference}

Acuna, B., J. Eliassen, J. Donoghue and J. Sanes (2002). "Frontal and parietal lobe activation during transitive inference in humans." Cereb Cortex 12(12): 1312-1321.

Alfred, K. L., A. C. Connolly and D. J. M. Kraemer (2018). "Putting the pieces together: Generating a novel representational space through deductive reasoning." Neuroimage 183: 99-111.

Ansari, D. (2008). "Effects of development and enculturation on number representation in the brain." Nat Rev Neurosci 9(4): 278-291.

Arsalidou, M., M. Pawliw-Levac, M. Sadeghi and J. Pascual-Leone (2018). "Brain areas associated with numbers and calculations in children: Meta-analyses of fMRI studies." Developmental Cognitive Neuroscience 30: 239-250.

Attout, L. and S. Majerus (2015). "Working memory deficits in developmental dyscalculia: The importance of serial order." Child Neuropsychol 21(4): 432-450.

Bryant, P. and H. Kopytynska (1976). "Spontaneous measurement by young children." Nature 260: 773.

Campbell, J. I. and Q. Xue (2001). "Cognitive arithmetic across cultures." J Exp Psychol Gen 130(2): 299-315.

Cantlon, J. F., E. M. Brannon, E. J. Carter and K. A. Pelphrey (2006). "Functional Imaging of Numerical Processing in Adults and 4-y-Old Children." PLoS Biology 4(5): e125.

Choi, H.-J., K. Zilles, H. Mohlberg, A. Schleicher, G. R. Fink, E. Armstrong and K. Amunts (2006). "Cytoarchitectonic Identification and Probabilistic Mapping of Two Distinct Areas within the Anterior Ventral Bank of the Human Intraparietal Sulcus." The Journal of Comparative Neurology 495(1): 53-69.

Chu, C., Y. Ni, G. Tan, C. J. Saunders and J. Ashburner (2011). "Kernel regression for fMRI pattern prediction." Neuroimage 56(2): 662-673.

Cognet, G. (2006). NEMI-2. Paris, France, ECPA.

De Visscher, A., A. Szmalec, L. Van Der Linden and M. P. Noel (2015). "Serial-order learning impairment and hypersensitivity-to-interference in dyscalculia." Cognition 144: 3848.

Eickhoff, S. B., K. E. Stephan, H. Mohlberg, C. Grefkes, G. R. Fink, K. Amunts and K. Zilles (2005). "A New SPM Toolbox for Combining Probabilistic Cytoarchitectonic Maps and Functional Imaging Data." NeuroImage 25(4): 1325-1335.

Emerson, R. W. and J. F. Cantlon (2015). "Continuity and change in children's longitudinal neural responses to numbers." Dev Sci 18(2): 314-326.

Evans, T. M., J. Kochalka, T. J. Ngoon, S. S. Wu, S. Qin, C. Battista and V. Menon (2015). "Brain structural integrity and intrinsic functional connectivity forecast 6 year longitudinal growth in children's numerical abilities." Journal of Neuroscience. 35(33): 11743-11750. Fangmeier, T., M. Knauff, C. C. Ruff and V. Sloutsky (2006). "FMRI evidence for a threestage model of deductive reasoning." J Cogn Neurosci 18(3): 320-334.

Feigenson, L., S. Dehaene and E. Spelke (2004). "Core systems of number." Trends Cogn Sci 8(7): 307-314.

Fias, W., J. Lammertyn, B. Caessens and G. A. Orban (2007). "Processing of Abstract Ordinal Knowledge in the Horizontal Segment of the Intraparietal Sulcus." The Journal of Neuroscience 27(33): 8952-8956.

Gabrieli, J. D. E., S. S. Ghosh and S. Whitfield-Gabrieli (2015). "Prediction as a Humanitarian and Pragmatic Contribution from Human Cognitive Neuroscience." Neuron 85(1).

Goel, V. and R. J. Dolan (2001). "Functional neuroanatomy of three-term relational reasoning." Neuropsychologia 39(9): 901-909. 
Goel, V. and R. J. Dolan (2003). "Explaining modulation of reasoning by belief." Cognition 87(1): B11-22.

Goodwin, G. P. and P. N. Johnson-Laird (2005). "Reasoning about relations." Psychol Rev 112(2): 468-493.

Handley, S., A. Capon, M. Beveridge, I. Dennis and J. S. B. T. Evans (2004). "Working memory, inhibitory control, and the development of children's reasoning." Thinking \& Reasoning 10: 175-195.

Hebart, M. N. and C. I. Baker (2018). "Deconstructing multivariate decoding for the study of brain function." Neuroimage 180: 4-18.

Heckers, S., M. Zalesak, A. Weiss, T. Ditman and D. Titone (2004). "Hippocampal activation during transitive inference in humans." Hippocampus 14(2): 153-162.

Hinton, E. C., S. Dymond, U. von Hecker and C. J. Evans (2010). "Neural correlates of relational reasoning and the symbolic distance effect: involvement of parietal cortex."

Neuroscience 168(1): 138-148.

Hoeft, F., B. D. McCandliss, J. M. Black, A. Gantman, N. Zakerani, C. Hulme, H. Lyytinen, S. Whitfield-Gabrieli, G. H. Glover, A. L. Reiss and J. D. Gabrieli (2011). "Neural systems predicting long-term outcome in dyslexia." Proc Natl Acad Sci U S A 108(1): 361-366. Hubbard, E. M., M. Piazza, P. Pinel and S. Dehaene (2005). "Interactions between number and space in parietal cortex." Nat Rev Neurosci 6(6): 435-448.

Inglis, M. and N. Attridge (2017). Does Mathematical Study Develop Logical Thinking?:

Testing the Theory of Formal Discipline. Singapore, World Scientific.

Inhelder, B. and J. Piaget (1958). The Growth of Logical Thinking from Childhood to Adolescence: An Essay on the Construction of Formal Operational Structures. New York, NY, Basic Books.

Knauff, M., T. Fangmeier, C. C. Ruff and P. N. Johnson-Laird (2003). "Reasoning, models, and images: behavioral measures and cortical activity." J Cogn Neurosci 15(4): 559-573.

Knauff, M., T. Mulack, J. Kassubek, H. R. Salih and M. W. Greenlee (2002). "Spatial imagery in deductive reasoning: a functional MRI study." Brain Res Cogn Brain Res 13(2): 203-212.

Knops, A. and K. Willmes (2014). "Numerical ordering and symbolic arithmetic share frontal and parietal circuits in the right hemisphere." Neuroimage 84: 786-795.

Lyons, I. M., G. R. Price, A. Vaessen, L. Blomert and D. Ansari (2014). "Numerical predictors of arithmetic success in grades 1-6." Dev Sci 17(5): 714-726.

Majerus, S., M. Poncelet, M. Van Der Linden, G. Albouy, E. Salmon, V. Sterpenich, G. Vandewalle, F. Collette and P. Maquet (2006). "The Left Intraparietal Sulcus and Verbal Short-Term Memory: Focus of Attention or Serial Order?" NeuroImage 32(2): 880-891. Marshuetz, C. (2005). "Order information in working memory: an integrative review of evidence from brain and behavior." Psychol Bull 131(3): 323-339.

Mathieu, R., J. R. Booth and J. Prado (2015). "Distributed neural representations of logical arguments in school-age children." Hum Brain Mapp 36(3): 996-1009.

Mathieu, R., J. Epinat-Duclos, J. Leone, M. Fayol, C. Thevenot and J. Prado (2018). "Hippocampal spatial mechanisms relate to the development of arithmetic symbol processing in children." Dev Cogn Neurosci 30: 324-332.

Mazaika, P., F. Hoeft, G. Glover and A. Reiss (2009). Methods and software for fMRI analysis for clinical subjects. The Organization of Human Brain Mapping, 15th Annual Meeting. San Francisco, CA, The Organization of Human Brain Mapping.

Mihalik, A., M. Brudfors, M. Robu, F. S. Ferreira, H. Lin, A. Rau, T. Wu, S. B. Blumberg, B. Kanber, M. Tariq, M. D. M. E. Garcia, C. Zor, D. I. Nikitichev, J. Mourao-Miranda and N. P. Oxtoby (2019). "ABCD Neurocognitive Prediction Challenge 2019: Predicting individual 
fluid intelligence scores from structural MRI using probabilistic segmentation and kernel ridge regression." arXiv:1905.10831 [q-bio, stat].

Morsanyi, K., A. Devine, A. Nobes and D. Szücs (2013). "The link between logic, mathematics and imagination: evidence from children with developmental dyscalculia and mathematically gifted children." Developmental science 16(4): 542-553.

Morsanyi, K., T. Kahl and R. Rooney (2017). The link between math and logic in adolescence: The effect of argument form. Individual differences in judgment and decision making from a developmental context. M. E. Toplak and J. Weller. Hove, UK, Hove: Psychology Press.: 166-185.

Morsanyi, K., T. McCormack and E. O'Mahony (2017). "The link between deductive reasoning and mathematics." Thinking \& Reasoning(1-24).

Moyer, R. S. and T. K. Landauer (1967). "Time required for judgements of numerical inequality." Nature 215(5109): 1519.

Mussolin, C., S. Mejias and M. P. Noel (2010). "Symbolic and nonsymbolic number comparison in children with and without dyscalculia." Cognition 115(1): 10-25.

Newman, S. D., G. Willoughby and B. Pruce (2011). "The effect of problem structure on problem-solving: an fMRI study of word versus number problems." Brain Res 1410: 77-88. Nieder, A. and S. Dehaene (2009). "Representation of number in the brain." Annu Rev Neurosci 32: 185-208.

Piaget, J. (1952). The child's conception of number. London, England, Routledge \& Kegan Paul.

Pinel, P., S. Dehaene, D. Riviere and D. LeBihan (2001). "Modulation of parietal activation by semantic distance in a number comparison task." Neuroimage 14(5): 1013-1026.

Pinel, P., M. Piazza, D. Le Bihan and S. Dehaene (2004). "Distributed and overlapping cerebral representations of number, size, and luminance during comparative judgments." Neuron 41(6): 983-993.

Portugal, L. C., M. J. Rosa, A. Rao, G. Bebko, M. A. Bertocci, A. K. Hinze, L. Bonar, J. R. Almeida, S. B. Perlman, A. Versace and C. Schirda (2016). "Can emotional and behavioral dysregulation in youth be decoded from functional neuroimaging?" PLoS one 11(1): e0117603.

Prabhakaran, V., B. Rypma and J. D. Gabrieli (2001). "Neural substrates of mathematical reasoning: a functional magnetic resonance imaging study of neocortical activation during performance of the necessary arithmetic operations test." Neuropsychology 15(1): 115-127. Prado, J., A. Chadha and J. R. Booth (2011). "The brain network for deductive reasoning: a quantitative meta-analysis of 28 neuroimaging studies." Journal of cognitive neuroscience 23(11): 3483-3497.

Prado, J., R. Mutreja and J. R. Booth (2013). "Fractionating the Neural Substrates of Transitive Reasoning: Task-Dependent Contributions of Spatial and Verbal Representations." Cereb Cortex 23(3): 499-507.

Prado, J., R. Mutreja, H. Zhang, R. Mehta, A. S. Desroches, J. E. Minas and J. R. Booth (2011). "Distinct representations of subtraction and multiplication in the neural systems for numerosity and language." Hum Brain Mapp 32(11): 1932-1947.

Prado, J., I. A. Noveck and J.-B. Van Der Henst (2010). "Overlapping and distinct neural representations of numbers and verbal transitive series." Cereb. Cortex 20(3): 720-729. Prado, J., J.-B. Van Der Henst and I. A. Noveck (2010). "Recomposing a fragmented literature: how conditional and relational arguments engage different neural systems for deductive reasoning." Neuroimage 51(3): 1213-1221.

Qin, S., S. Cho, T. Chen, M. Rosenberg-Lee, D. C. Geary and V. Menon (2015). "Hippocampal-neocortical functional reorganization underlies children's cognitive development." Nature Neuroscience 17(9): 1263-1269. 
Rabinowitz, F. M. and M. L. Howe (1994). "Development of the middle concept." Journal of Experimental Child Psychology 57: 418-449.

Scheperjans, F., S. B. Eickhoff, L. Homke, H. Mohlberg, K. Hermann, K. Amunts and K. Zilles (2008). "Probabilistic Maps, Morphometry, and Variability of Cytoarchitectonic Areas in the Human Superior Parietal Cortex." Cerebral Cortex 18(9): 2141-2157.

Scheperjans, F., K. Hermann, S. B. Eickhoff, K. Amunts, A. Schleicher and K. Zilles (2008). "Observer-Independent Cytoarchitectonic Mapping of the Human Superior Parietal Cortex." Cerebral Cortex 18(4): 846-867.

Schrouff, J., M. J. Rosa, J. M. Rondina, A. F. Marquand, C. Chu, J. Ashburner, C. Phillips, J. Richiardi and J. Mourão-Miranda (2013). "PRoNTo: Pattern Recognition for Neuroimaging Toolbox." Neuroinformatics 11(3): 319-337.

Schwartz, F., J. Epinat-Duclos, J. Leone, A. Poisson and J. Prado (2018). "Impaired neural processing of transitive relations in children with math learning difficulty." Neuroimage Clin 20: $1255-1265$.

Schwartz, F., J. Epinat-Duclos, J. Léone, A. Poisson and J. Prado (2018). "Impaired neural processing of transitive relations in children with math learning difficulty." NeuroImage: Clinical 20: 1255-1265.

Schwartz, F., J. Epinat-Duclos, J. Léone, A. Poisson and J. Prado (2018). "Impaired neural processing of transitive relations in children with math learning difficulty." NeuroImage: Clinical.

Schwartz, F., J. Epinat-Duclos, J. Leone and J. Prado (2017). "The neural development of conditional reasoning in children: Different mechanisms for assessing the logical validity and likelihood of conclusions." Neuroimage 163: 264-275.

Shawe-Taylor, J. and N. Cristianini (2004). Kernel Methods for Pattern Analysis, Cambridge University Press.

Singley, A. T. M. and S. A. Bunge (2014). "Neurodevelopment of relational reasoning: Implications for mathematical pedagogy." Trends in Neuroscience and Education 3(2): 33-37. Smith, S. M. and T. E. Nichols (2009). "Threshold-free cluster enhancement: addressing problems of smoothing, threshold dependence and localisation in cluster inference." Neuroimage 44(1): 83-98.

Sokolowski, H. M., W. Fias, A. Mousa and D. Ansari (2017). "Common and distinct brain regions in both parietal and frontal cortex support symbolic and nonsymbolic number processing in humans: A functional neuroimaging meta-analysis." Neuroimage 146: 376-394. Supekar, K., A. G. Swigart, C. Tenison, D. D. Jolles, M. Rosenberg-Lee, L. Fuchs and V. Menon (2013). "Neural predictors of individual differences in response to math tutoring in primary-grade school children." Proc Natl Acad Sci U S A 110(20): 8230-8235.

Taylor, J. A., N. Matthews, P. T. Michie, M. J. Rosa and M. I. Garrido (2017). "Auditory prediction errors as individual biomarkers of schizophrenia." NeuroImage: Clinical 15: 264273.

Thevenot, C., P. Barrouillet, C. Castel and K. Uittenhove (2016). "Ten-year-old children strategies in mental addition: A counting model account." Cognition 146: 48-57.

Vogel, S. E., C. Goffin and D. Ansari (2015). "Developmental specialization of the left parietal cortex for the semantic representation of Arabic numerals: an fMR-adaptation study." Dev Cogn Neurosci 12: 61-73.

Vul, E., C. Harris, P. Winkielman and H. Pashler (2009). "Puzzlingly high correlations in fMRI studies of emotion, personality, and social cognition." Perspect Psychol Sci 4: 274-290. Wendelken, C. (2015). "Meta-analysis: how does posterior parietal cortex contribute to reasoning?" Frontiers in human neuroscience 8(1042).

Wertheim, J. and M. Ragni (2018). "The Neural Correlates of Relational Reasoning: A Metaanalysis of 47 Functional Magnetic Resonance Studies." J Cogn Neurosci 30(11): 1734-1748. 
Woodcock, R. W., K. S. McGrew and N. Mather (2001). Woodcock-Johnson III Tests of Achievement. Itasca, IL, The Riverside Publishing Company.

Wright, B. C. (2001). "Reconceptualizing the transitive inference ability: A framework for existing and future research." Developmental Review 21(4): 375-422.

Zamarian, L., A. Ischebeck and M. Delazer (2009). "Neuroscience of learning arithmetic-evidence from brain imaging studies." Neurosci Biobehav Rev 33(6): 909-925. 


\section{Figures}

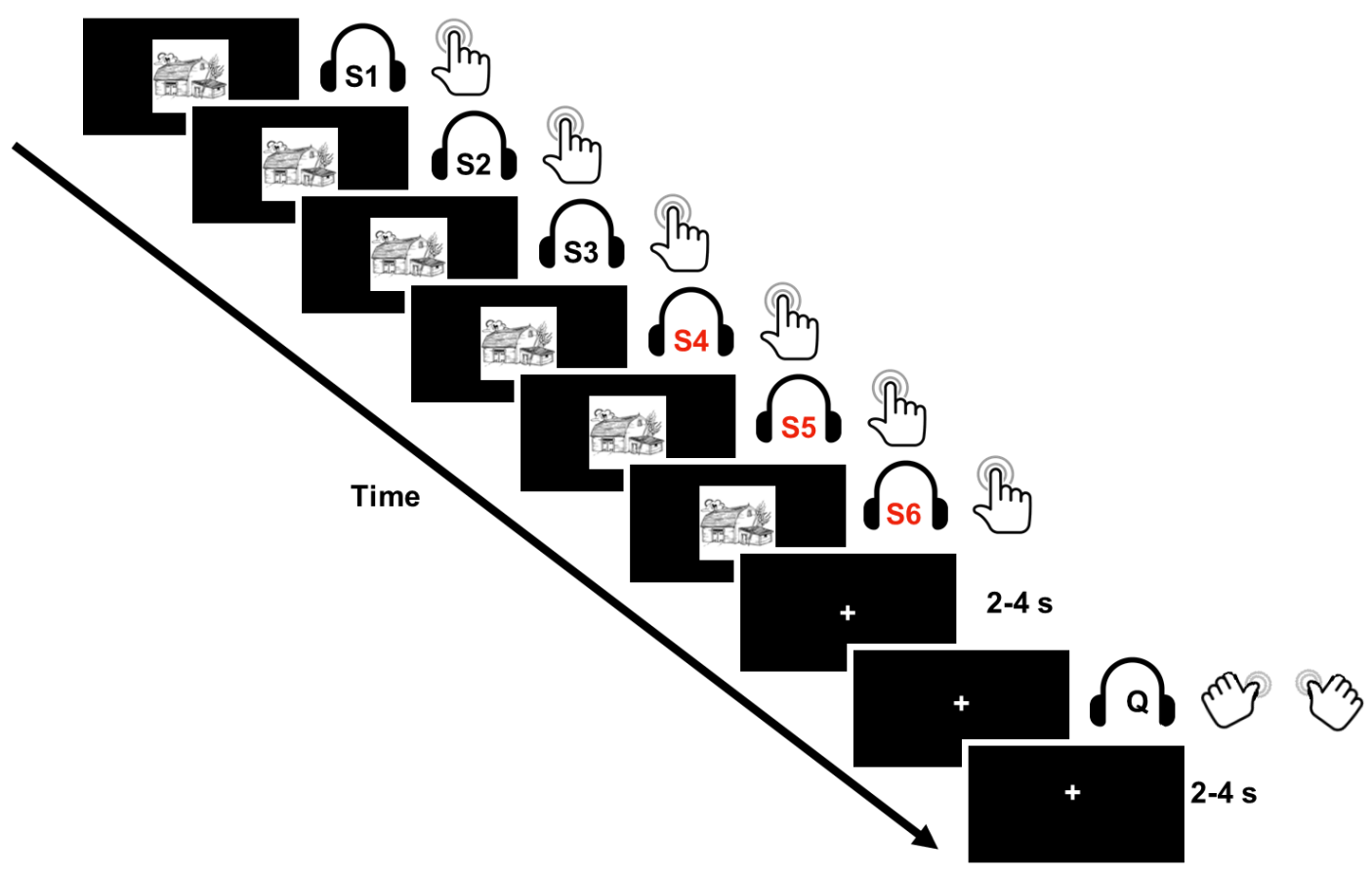

Figure 1. Timeline for a sample scenario. Each of the 6 sentences (S) was spoken through headphones while a picture was displayed on the screen. The task was entirely self-paced. Participants pressed on a button to indicate that they were ready to listen to the next sentence, which was spoken after a $500 \mathrm{~ms}$ delay (not shown). The scenario ended with a question (Q), which was also spoken through headphones. This question was preceded and followed by a jittered interval ranging from 2 to 4 seconds. The sentences of interest considered in the analyses were sentences 4 to 6 (in red). 

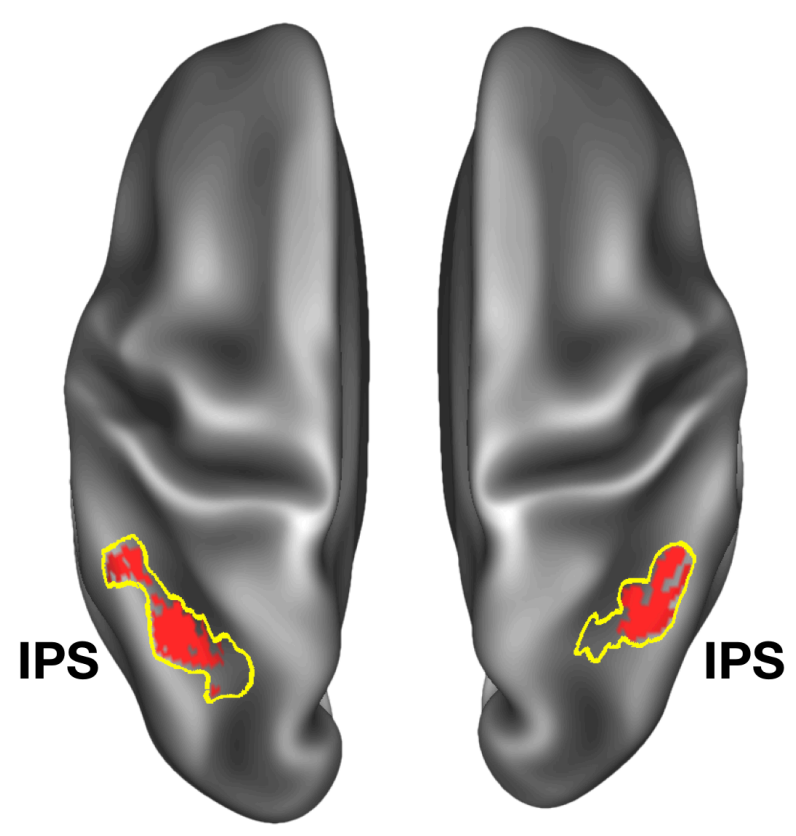

Figure 2. Brain regions involved in transitive reasoning across all participants. Clusters in red are regions in which greater activity was observed for transitive than non-transitive relations. Yellow outlines delineate the IPS mask used for small volume correction (see Methods). Activations are overlaid on an inflated 3D rendering of the MNI-normalized anatomical brain (lateral views of the left and right hemispheres). 
A

Calculation

L. IPS

R. IPS

L. IPS

R. IPS

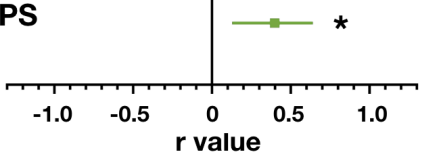

B

Calculation

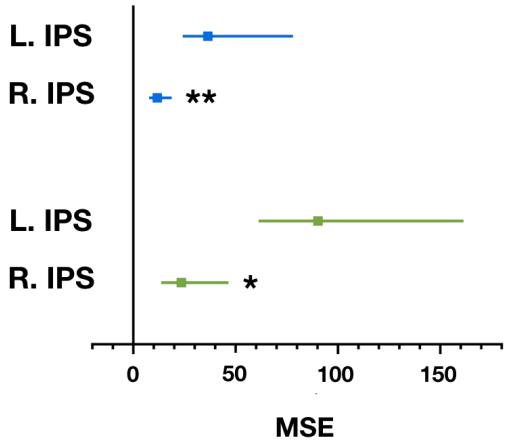

Math fluency

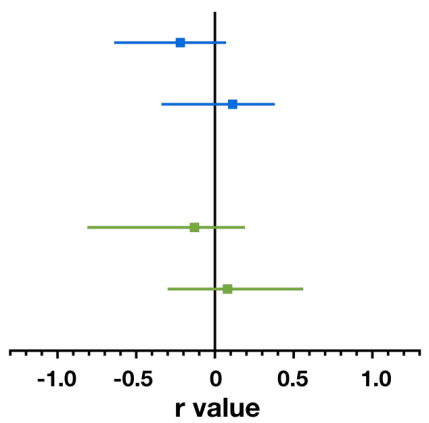

Math fluency

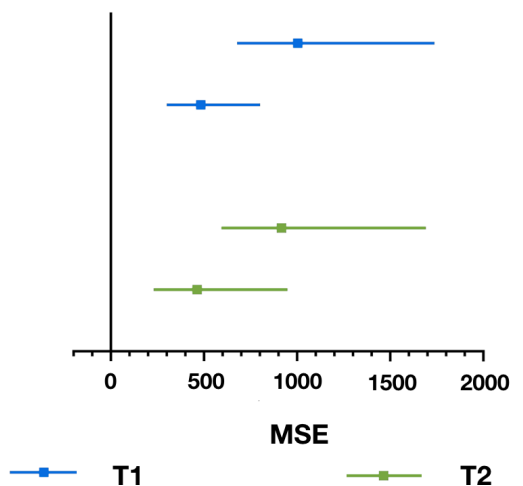

Applied problems

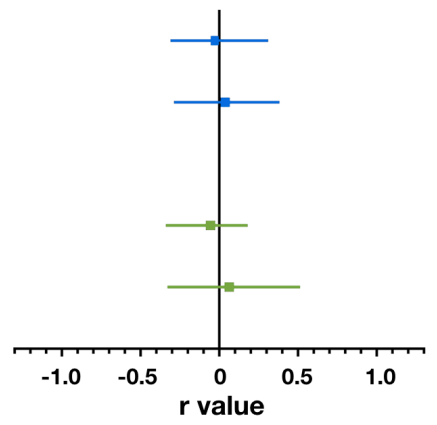

Applied problems

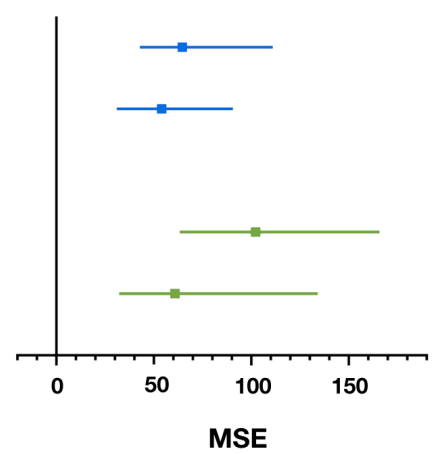

Figure 3. Results of multivariate analyses. (A) Pearson's correlation coefficient (r) for the relationship between actual and predicted scores broken down by subtest (Calculation, Math fluency, and Applied problems) and time of testing $\left(\mathrm{T}_{1}, \mathrm{~T}_{2}\right)$. Error bars represent bootstrapped 95\% confidence intervals. (B) Mean squared error (MSE) for the relationship between actual and predicted scores broken down by subtest (Calculation, Math fluency, and Applied problems) and time of testing $\left(T_{1}, T_{2}\right)$. Error bars represent bootstrapped $95 \%$ confidence intervals. *, $\mathrm{p}<0.05 ; * *, \mathrm{p}<0.01$; L., left; R., right. 


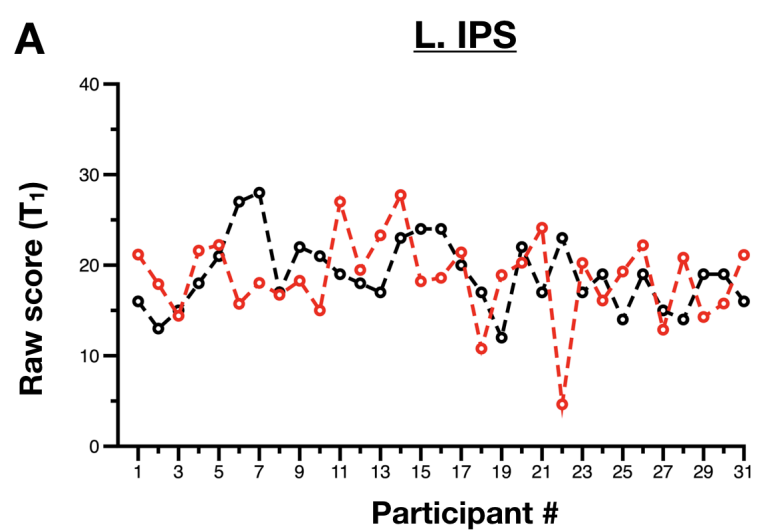

B

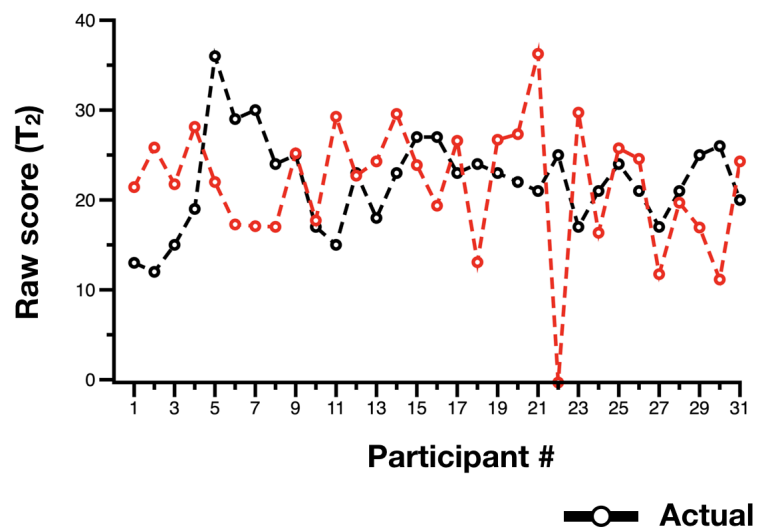

R. IPS

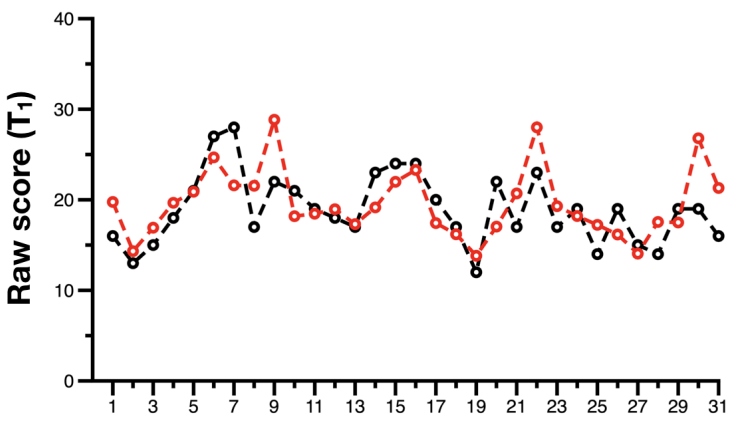

Participant \#

R. IPS

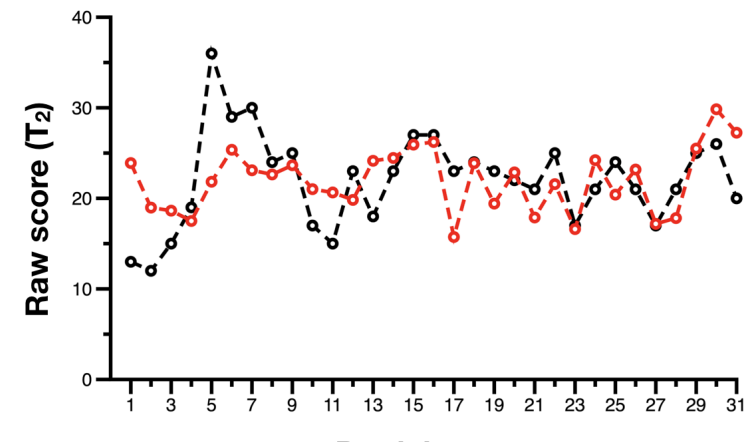

Participant \#

Figure 4. Individual predictions of Calculation scores. (A) Line plots showing the consistency between the actual and the predicted Calculation score for each participant in the left and right IPS at $\mathrm{T}_{1}$. (B) Line plots showing the consistency between the actual and the predicted Calculation score for each participant in the left and right IPS at $\mathrm{T}_{2}$. 


\section{Tables}

Table 1. Demographic information and measures of verbal IQ, reasoning (matrices) abilities and math skills.

\section{Variable}

Demographic information

Age at $T_{1}$ (in years)

Age at $\mathrm{T}_{2}$ (in years)

Verbal IQ (NEMI-II)

General knowledge ${ }^{1}$

Vocabulary $^{1}$

Comparison $^{1}$

Matrix reasoning (NEMI-II)

Raven's matrices ${ }^{1}$

Math skills (WJ-III)

Calculation $\left(\mathrm{T}_{1}\right)^{2}$

Math fluency at $\left(\mathrm{T}_{1}\right)^{2}$

Applied problems at $\left(\mathrm{T}_{1}\right)^{2}$

Calculation $\left(\mathrm{T}_{2}\right)^{2}$

Math fluency at $\left(\mathrm{T}_{2}\right)^{2}$

Applied problems at $\left(\mathrm{T}_{2}\right)^{2}$
$105.85(20.85)$

\section{Mean (SD)}

$10.97(1.37)$

$12.57(1.39)$

$105.85(20.4)$

$106.75(18.45)$

$112.15(21.75)$

$18.90(3.82)$

$51.16(17.43)$

$37.23(5.33)$

$22.03(5.18)$

$62.97(16.36)$

$40.03(6.04)$ 
Table 2. Examples of each type of scenario (translated from French)

\begin{tabular}{|c|c|c|}
\hline & Transitive relations & Non-transitive relations \\
\hline 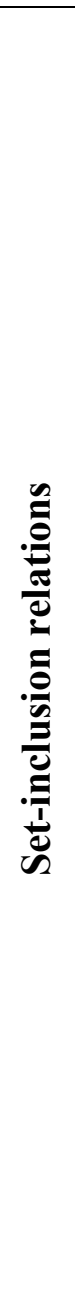 & $\begin{array}{l}\text { 1. "You are going on vacation to the } \\
\text { countryside." } \\
\text { "You are planning to stay in a farm for a } \\
\text { few days." } \\
\text { "There are farms uphill and downhill." } \\
\text { "All old farms are made of stone." } \\
\text { "All farms that are made of stone are } \\
\text { uphill." } \\
\text { "You have to find an old farm." } \\
\text { Reasoning question: "Are you going } \\
\text { uphill (response 1) or downhill (response } \\
\text { 2)?" }\end{array}$ & $\begin{array}{l}\text { 2. "You are going uphill and you find the old farm. } \\
\text { (response 1)" / "You are going downhill and the } \\
\text { farmers pick you up" (response 2) } \\
\text { "The farmers invite you in." } \\
\text { "You need to bring your bag to your bedroom on the } \\
\text { 2nd floor." } \\
\text { "All bedrooms with a red door are next to the } \\
\text { chicken coop." } \\
\text { "All bedrooms with a green door are next to the } \\
\text { barn." } \\
\text { "The farmers' house is very big." } \\
\text { Memory question: "Are you taking your bag to the } \\
3^{\text {rd }} \text { floor (response 1) or to the } 2^{\text {nd }} \text { floor (response } \\
\text { 2)?", }\end{array}$ \\
\hline
\end{tabular}




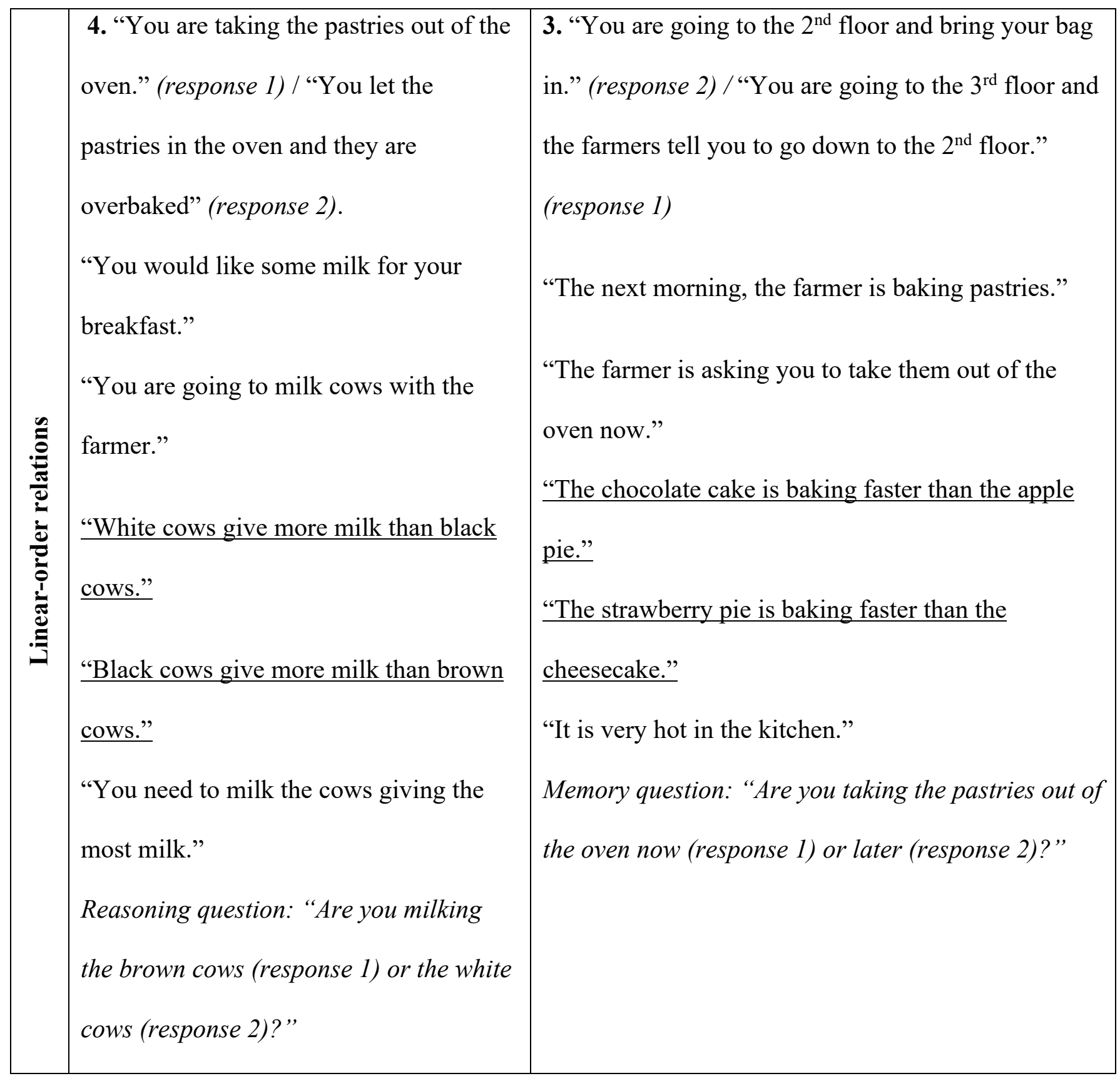

Notes. Numbers 1 to 4 indicate the order of presentation within the experimental run. 
Table 3. Clusters activated in the contrast of transitive versus non-transitive relations across all participants

\section{MNI coordinates}

Whole-brain SVC

Cluster size

\begin{tabular}{lccccccc} 
Anatomical location & PFWE-corr & PFWE-corr & X & Y & Z & t-score & $\left(\mathrm{mm}^{3}\right)$ \\
\hline Left hIP1/hIP2 & 0.102 & 0.002 & -46 & -46 & 38 & 4.88 & 644 \\
Left hIP1/hIP3 & 0.226 & 0.007 & -32 & -56 & 41 & 3.57 & 1,568 \\
Right hIP1/hIP2/hIP3 & 0.368 & 0.004 & 42 & -48 & 41 & 4.03 & 2,156
\end{tabular}

Notes. BA: Brodmann area; MNI: Montreal Neurological Institute; SVC: Small Volume Correction;

FWE-corr: Family-wise error corrected. 
Table 4. Partial correlation matrix of math scores, performance on the transitive reasoning task, and univariate IPS activity, controlling for age and verbal IQ (General knowledge, Vocabulary, Comparison).

\begin{tabular}{|c|c|c|c|c|c|c|c|c|c|c|}
\hline & 1 & 2 & 3 & 4 & 5 & 6 & 7 & 8 & 9 & 10 \\
\hline 1- Calculation (T1) & - & & & & & & & & & \\
\hline 2- Math fluency (T1) & $0.50 * *$ & - & & & & & & & & \\
\hline 3- Applied problems (T1) & $0.47 * *$ & $0.76 * * *$ & - & & & & & & & \\
\hline 4- Calculation (T2) & $0.49 * *$ & $0.49 * *$ & $0.62 * * *$ & - & & & & & & \\
\hline 5- Math fluency (T2) & $0.31 \dagger$ & $0.74 * * *$ & $0.72 * * *$ & $0.68 * * *$ & - & & & & & \\
\hline 6- Applied problems (T2) & 0.30 & $0.64 * * *$ & $0.81 * * *$ & $0.71 * * *$ & $0.73 * * *$ & - & & & & \\
\hline 7- Reasoning (accuracy) & 0.27 & $0.38 *$ & $0.42 *$ & 0.12 & 0.17 & 0.14 & - & & & \\
\hline 8- Memory (accuracy) & $0.41 *$ & 0.11 & 0.10 & -0.01 & 0.02 & -0.05 & $0.47 * *$ & - & & \\
\hline 9- Left IPS (beta) & $0.41 *$ & -0.02 & 0.06 & $0.50 * *$ & 0.09 & 0.10 & 0.14 & -0.01 & - & \\
\hline 10- Right IPS (beta) & $0.34 \dagger$ & 0.02 & -0.09 & $0.41 *$ & 0.04 & -0.07 & 0.07 & -0.12 & $0.84 * * *$ & - \\
\hline
\end{tabular}

Notes. $\uparrow, \mathrm{p}<0.1, * \mathrm{p}<0.05, * * \mathrm{p}<0.01, * * * \mathrm{p}<0.001$ 